\title{
Efecto de la asistencia técnica en el desempeño profesional
} Effect of technical assistance
on professional performance Efeito da assistência técnica no desempenho profissional

\author{
Milagros Castillo-Fuerman' \& \\ https://orcid.org/0000-0001-8039-1547 \\ Herbert Robles Mori \\ https://orcid.org/0000-0002-5937-5937
}

https://doi.org/10.53287/mkfb8365en84z

Fecha de ingreso: 11 de noviembre de 2020

Fecha de aprobación: 17 de julio de 2021

Conflictos de interés: Los autores declaran no tener conflictos de intereses.

\section{RESUMEN}

La presente investigación tuvo la finalidad de identificar las ventajas de la asistencia técnica en los procesos de formación continua y sus efectos en el desempeño de profesionales de salud que atienden a niños y niñas menores de 3 años en el Control de Crecimiento y Desarrollo (CRED). La investigación evidenció que al incorporar la variable Asistencia Técnica (AT), los programas de formación continua son más eficientes, es decir, cuando se fortalecen capacidades en el campo de la acción

1 - Milagros Castillo Fuerman. Magister en Educación con mención en Docencia e Investigación en Educación Superior, Profesora de Educación Inicial con Especialización en Planeamiento Estratégico del sector público, Diploma en investigación Cualitativa, Diploma clínico de evaluación e intervención en infancia. Asociada Senior de Programas en Desarrollo Infantil temprano de UNICEF, Miembro asociado de Foro Educativo y Docente de la Universidad Peruana Cayetano Heredia. Email: milagros.castillo.f@upch.pe; milicastillo29@yahoo.com

- Asesor: Dr. Herbert Robles Mori. Doctor en Ciencias de la Educación, Magister en Psicología con mención en Diagnóstico e Intervención Psicoeducativa, Magister en educación con mención en problemas de Aprendizaje, Licenciado en Psicología, Catedrático de la Universidad Femenina del Sagrado Corazón, Universidad Peruana Cayetano Heredia y Universidad San Ignacio de Loyola. Email: hrobles@unife.edu.pe; Herbert.robles.m@upch.pe; 
profesional. El diseño del estudio fue cuantitativo, según su profundidad es explicativo de tipo cuasiexperimental, orientado a identificar la relación de causalidad o efecto de la asistencia técnica en el desempeño profesional, comparando un grupo experimental y un grupo control. Los resultados generales muestran diferencias significativas, el grupo experimental, alcanzó una media del 51.67, mientras que el grupo control solo llegó a 26.53, aplicando la prueba no paramétrica U Mann Whitney: $p \leq .01$. Si bien la formación continua genera avances en ambos grupos, al incorporar la variable AT se evidencia un salto significativo respecto al grupo control, concluyendo que la asistencia técnica mejora el desempeño del personal de salud que atienden en el Control de CRED, fortaleciendo e incrementando sus capacidades.

\section{PALABRAS CLAVE}

Asistencia técnica; Desempeño profesional; Desarrollo infantil temprano; Formación continua; Control de CRED.

\section{ABSTRACT}

The purpose of this research was to identify the advantages of technical assistance in continuous training processes and its effects on the performance of health professionals who care for children under 3 years of age in the Growth and Development Control (CRED). The research showed that by incorporating the Technical Assistance (TA) variable, continuous training programs are more efficient, that is, when capacities are strengthened in the field of professional action. The study design was quantitative, according to its depth it is explanatory of a quasi-experimental type, aimed at identifying the causal relationship or effect of technical assistance on professional performance, comparing an experimental group and a control group. The general results show significant differences, the experimental group, reaching an average of 51.67, while the control group only reached 26.53 , applying the non-parametric Mann Whitney $U$ test: $p \leq .01$. Although continuous training generates progress in both groups, when incorporating the AT variable, a significant jump is evidenced compared to the control group, concluding that technical assistance improves the performance of the health personnel who attend to the CRED Control, strengthening and increasing their capabilities.

\section{KEYWORDS}

Technical assistance; Professional performance; Early childhood development; Continuous training; CRED control.

\section{RESUMO}

O objetivo desta pesquisa foi identificar as vantagens da assistência técnica nos processos de formação contínua e seus reflexos na atuação dos profissionais de saúde que cuidam de crianças menores de 3 anos no Controle de Crescimento e Desenvolvimento (CRED). A pesquisa mostrou que, ao incorporar a variável Assistência Técnica (AT), os programas de formação contínua são mais eficientes, isto é, quando se fortalecem as capacidades no campo da atuação profissional. O delineamento do estudo foi quantitativo, pois pela sua profundidade é explicativo do tipo quase experimental, visando identificar a relação causal ou efeito da assistência técnica no desempenho profissional, comparando um grupo experimental e um grupo controle. Os resultados gerais mostram diferenças significativas, o grupo experimental, atingindo a média de 51,67, enquanto o grupo controle atingiu apenas 26,53, aplicando-se o teste não paramétrico $U$ de Mann Whitney: $p \leq, 01$. Embora o 
treinamento contínuo gere avanços em ambos os grupos, ao incorporar a variável TA, evidencia-se um salto significativo em relação ao grupo controle, concluindo que a assistência técnica melhora o desempenho dos profissionais de saúde que atendem ao Controle CRED, fortalecendo e aumentando sua capacidades.

\section{PALAVRAS-CHAVE}

Assistência técnica; Atuação profissional; Desenvolvimento infantil; Formação contínua; Controle CRED.

\section{Introducción}

En los últimos tiempos las estrategias de formación profesional están orientadas a mejorar el desempeño y no solo a incrementar conocimientos. Las diferentes tendencias y enfoques en cuanto a la formación continua de profesionales coinciden en que no es suficiente una preparación académica, además, se requiere de un adecuado entrenamiento de habilidades que fortalezcan el desempeño profesional. De allí la necesidad de indagar sobre estrategias de acompañamiento o asistencia técnica enfocadas en la mejora de aprendizajes en los servicios donde trabajan.

De acuerdo a lo señalado por Tejedor (2019) citando a Salas-Perea et al. (2016) las competencias y el desempeño son parte de "un conjunto de condiciones laborales y personales requeridas para su actuación en un determinado puesto de trabajo" (p.3). Salas-Perea et al. (2016), agregan que la calificación profesional ya no es concebida solo como la acumulación de saberes o habilidades, sino como la capacidad de actuar, intervenir y decidir en situaciones no siempre previstas; cambiando así el foco de atención, de la calificación académica, al logro de competencias en el desempeño profesional.

En el Perú, las experiencias de asistencia técnica orientadas a la mejora del desempeño profesional han estado más visibilizadas en profesionales de educación. La mayoría de los programas nacionales de formación continua promovidos des- de el Ministerio de Educación (Minedu), se complementaron con programas de acompañamiento pedagógico o asistencia técnica al desempeño del docente. Adicionalmente, se implementó la estrategia de formación continua denominada acompañamiento pedagógico, cuyos resultados han evidenciado cambios positivos en el rendimiento académico de los estudiantes, como muestran investigaciones (Rodríguez et al., 2016; Salluca y Valeriano, 2018; Balarin y Escudero, 2019).

En el caso del sector salud, las experiencias de formación han sido focalizadas o especializadas, no se evidencian programas nacionales de capacitación continua, complementadas con asistencia técnica desarrolladas por el Ministerio de Salud (Minsa) o la Escuela Nacional de Salud Pública. Las capacitaciones a profesionales que atienden en el CRED eran prioritariamente orientadas a acciones preventivas de salud, antropometría, estado nutricional, lactancia o inmunizaciones, con soportes de supervisión más que de acompañamiento; mientras que los temas vinculados al desarrollo infantil, evaluación o estimulación temprana, eran menos abordados. Por ello, la necesidad de identificar estrategias de formación continua que contribuyan con una capacitación más integral, orientada a la atención temprana y que incorpore el acompañamiento en servicio para mejorar el desempeño del personal de salud.

Por ello, el presente estudio tuvo la finalidad de contar con evidencias que 
muestren la efectividad de la asistencia técnica en profesionales de los servicios de salud del CRED que participan de un programa de formación continua, para visibilizar estrategias efectivas que contribuyan con la mejora del desempeño de los profesionales que atienden a los niños y niñas en edades tempranas y sus familias. Planteándose la siguiente pregunta de investigación ¿Cuál será el efecto de la asistencia técnica sobre el desempeño de profesionales de salud que participan en el programa de formación continua en Desarrollo infantil temprano implementado por una universidad pública?

El estudio describe las estrategias, instrumentos y características de la asistencia técnica orientada al personal de salud en las acciones de vigilancia y promoción del desarrollo de niñas y niños de 0 a 3 años en habilidades y destrezas cognitivas, físicas, afectivas y sociales, con el fin de contribuir con la calidad del servicio de salud integral que se brinda. Se centró en aspectos del desempeño del personal de salud para favorecer el desarrollo infantil temprano (DIT) en el CRED, más no en aspectos de crecimiento o estado nutricional; porque fue el contenido principal del programa de formación en DIT.

Es relevante señalar la importancia del estudio, pues busca generar evidencia sobre los efectos de la AT en el desempeño de profesionales que participan en procesos de formación continua. Así aportar en el diseño de estrategias e instrumentos que permitan asistir a profesionales de la salud con mayor eficiencia, para mejorar su desempeño y contribuir con la calidad de los servicios que brindan en la promoción de un desarrollo adecuado de niños y niñas menores de 3 años y el acompañamiento a sus familias. Además, está orientada a aportar en la mejora de la calidad de los programas de formación continua a profesionales de salud que atienden en servicios de infancia temprana.
Los resultados del estudio demuestran que cuando los procesos de formación continua combinan espacios académicos con asistencia técnica en sus servicios, se logra mayor efectividad en el desempeño profesional. Los participantes del programa de formación en DIT que complementaron las sesiones de capacitación con visitas de asistencia técnica en sus establecimientos de salud, alcanzaron un mejor desempeño, respecto a los que solo participaron en las sesiones de capacitación, encontrando diferencias significativas en su capacidad para generar mejores interacciones con el niño o niña en la atención del Control de CRED, al brindar consejería familiar, aplicar la evaluación del desarrollo y al organizar las condiciones de espacio, tiempo y recursos que requiere el servicio.

\section{La asistencia técnica y el desempe- ño profesional}

La asistencia técnica, es el conjunto de actividades orientadas al fortalecimiento de capacidades en el ejercicio del desempeño profesional, que promueven aprendizajes significativos, participación protagónica y buscan conectar la teoría con la práctica. Esto genera procesos de autorreflexión y compromiso en quien aprende para proponer cambios que contribuyan con la mejora continua de su propio desempeño y en la gestión eficiente de los servicios que brinda. Es asistido por un profesional de mayor experiencia quien asume el rol de facilitador o mediador de aprendizajes, genera procesos de análisis, toma de decisiones, autonomía y compromiso (Minedu, 2014; Minedu, 2016; Rodríguez et al., 2016; (Balarin y Escudero, 2019).

Según la experiencia de Asistencia técnica, Cooperación y Acompañamiento Territorial Minsalud (2014) señalan que "la asistencia técnica es un proceso orientado a mejorar las capacidades en las personas, la territorial y los resultados mediante 
asesoría, cooperación- acompañamiento técnico y capacitación, en trabajo conjunto con los actores involucrados". (p. 16). Agregan que este proceso debe partir de la identificación de necesidades, respeto a la autonomía, reconocer las competencias existentes, para generar espacios de concertación y la transformación sostenible de los servicios de salud.

De acuerdo a la Norma Técnica de Salud del CRED (Minsa, 2017) se redefine las acciones de supervisión en acciones de asistencia técnica orientadas a la mejora del desempeño y la calidad del control del crecimiento y desarrollo en los establecimientos de salud, lo que implica el desarrollo de acciones de acompañamiento, la misma que se define como un proceso de enseñanza-aprendizaje, en el que se interactúa con el potencial humano supervisado, analizando los elementos críticos identificados a fin de implementar medidas correctivas (p. 27).

La supervisión generalmente ha tenido una carga fiscalizadora, que no siempre contribuye con un cambio actitudinal y mejora del desempeño. En tal sentido, la concepción que presenta la NTS del CRED, representa una gran oportunidad para generar nuevos estilos de acompañamiento al personal de salud que, además, están vinculados con los procesos de formación y promoción de aprendizajes, desde un sentido crítico que genere cambios en su desempeño.

El desempeño profesional se define como la conducta real de los trabajadores, tanto en el orden profesional, como en las relaciones interpersonales que se crean en la atención integral de salud. La calidad del desempeño está unida e integrada a la calidad de los servicios (Salas-Perea et al., 2016; Torres-López et al., 2017).

El desempeño profesional, es el ejercicio de las funciones y el rol que asume en el campo laboral, en el que se evidencia sus saberes, concepciones, enfoques, creencias, habilidades, procedimientos y actitudes, que suponen una actuación real en el contexto en el que laboran y en el que logra demostrar sus competencias profesionales (Minedu, 2016; Rodríguez et al., 2016; Solís et al., 2019).

Al analizar el desempeño profesional, Torres-López et al. (2017) explica la relación de las competencias laborales con la idoneidad demostrada que se evidencia sus en el ejercicio de las funciones, la cuales están vinculada con un conjunto de conocimientos, habilidades, experiencias, sentimientos, actitudes, motivaciones, características personales y valores. Estos deben ser observables y medibles, deben contribuir al logro de los objetivos de la organización a la que pertenecen, demostrando un rendimiento y actuación, de acuerdo con los requerimientos establecidos para su cargo.

Al establecer las dimensiones del estudio, se seleccionaron los aspectos de estructura y de proceso que están vinculados al desempeño profesional. Monteiros (2015) utiliza los aspectos propuestos por a Donabedian (1990) para definir los criterios de su investigación sobre la satisfacción del usuario en el control de salud infantil en un centro de salud familiar; considerando: (1) la estructura, que concierne a los aspectos físicos, equipos, materiales, recursos; (2) el proceso, que comprende todo lo que los profesionales de la salud realizan por los pacientes y sus habilidades profesionales; y (3) los resultados, que comprenden los cambios en el estado de salud y se miden a partir de los objetivos propuestos en la intervención.

López et al. (2016) citan a Thomason y La Paro (2009) para explicar las variables estructurales y de proceso. Definen las variables estructurales a la presencia o ausencia de aquellos recursos que facili- 
tan la atención o las características del entorno y son además variables que pueden ser reguladas con mayor facilidad. Por su parte, las variables de proceso tienden a focalizarse en aspectos dinámicos como las interacciones que los niños o niñas tienen con sus cuidadores, la relación con las familias, el comportamiento de los cuidadores, entre otros aspectos.

El estudio cualicuantitativo sobre la atención de salud de niños menores de tres años en nueve regiones con altos índices de pobreza en Perú, realizado por Cordero et al. (2016) evaluó los servicios de CRED en 18 establecimientos de salud. Se identificaron aspectos de proceso y estructura vinculados a cinco elementos importantes en la calidad de la atención: espacio físi$c o$, equipamiento, personal, tiempo para la atención y consejería.

Las dimensiones del presente estudio identificaron aspectos de estructura y de proceso que están vinculados al desempeño profesional. Entendiendo las dimensiones de estructura, como la capacidad para organizar las condiciones físicas y materiales para la atención. Y las de proceso, como la capacidad para la aplicación de técnicas y procedimientos, manteniendo interacciones positivas en la atención con niños y niñas y sus familias.

En el caso de estructura, se consideraron dos componentes: la capacidad para organizar las condiciones de espacio y para seleccionar recursos o materiales. Mientras que, en el caso de proceso, se consideraron tres componentes: capacidad para mantener interacciones positivas con niños y niñas, capacidad técnica para aplicar los instrumentos de evaluación del desarrollo y capacidad para brindar consejería a la familia en DIT. La presente investigación no realiza el análisis de los resultados en los niños y niñas, lo cual puede ser objeto de otra investigación; centró el estudio en dimensiones vinculadas al desempeño del personal de salud.
Las dimensiones de estructura y proceso del desempeño se organizaron en cinco, para efectos de esta investigación:

- Dimensión 1: Organiza el espacio para promover Desarrollo Infantil Temprano (DIT) en el Control de CRED.

- Dimensión 2: Organiza recursos y materiales para promover DIT.

- Dimensión 3: Interacciones del personal de salud con el niño o niña durante la atención en el Control de CRED.

- Dimensión 4: Evalúa el desarrollo infantil en el Control de CRED considerando las necesidades y condiciones físicas y afectivas del niño o niña.

- Dimensión 5: Consejería a la familia durante la atención en el Control de CRED.

La asistencia técnica en el Programa de formación continua, desarrollada en esta investigación, propone visitas de AT al personal de salud del CRED que recibieron la capacitación en DIT. Es realizada por el equipo de capacitadores o asistentes técnicos y se espera que estos espacios sean formativos, no de evaluación o monitoreo, sino de reflexión sobre el desempeño en la práctica. Por ello, parten de la observación en la atención del personal de salud en el CRED, para luego generar un momento de revisión colectiva de un instrumento que describe el desempeño. A partir de esa revisión se movilizan procesos de autoreflexión orientados a fortalecer capacidades.

\section{Método}

\subsection{Participantes}

La población de estudio estuvo constituida por todas las enfermeras que atienden en el control del CRED que participaron del programa de formación continua en DIT en una universidad estatal en la ciudad de Ayacucho. 
La unidad de análisis es una enfermera que atienden en el Control de CRED que participaron del programa de formación continua en DIT.

Con respecto a la muestra, se trabajó con el total poblacional (30 profesionales de enfermería); por lo tanto, no se determinó un tamaño de muestra. Se incluyen a todos los participantes del programa de Formación en DIT, considerando todos los recursos humanos disponibles para esta investigación.

La muestra No probabilística disponible e intencional, puede considerar a toda la población cuando el tamaño es pequeño o tiene una población determinada o limitada que está al alcance del investigador (Otzen y Manterola, 2017; Neill y Cortez, 2018). En este caso, la totalidad de la población estaba disponible y fueron divididos aleatoriamente en dos grupos.

La conformación del grupo de estudio fue una población de 30 profesionales de enfermería que se dividió en forma aleatoria para conformar el grupo control (15) y el grupo experimental (15), con la ayuda de un programa informático de Excel.

\subsection{Tipo y diseño de investigación}

El diseño corresponde al paradigma de la investigación cuantitativa, es explicativa de tipo cuasiexperimental. Según el objetivo de la investigación es de tipo aplicada y según su profundidad es de nivel explicativo, porque busca la causalidad o la explicación de una variable sobre otra (Morales, 2012; Neill, D. y Cortez, 2018).

El tratamiento de la información muestra el efecto de la AT en el desempeño profesional, comparando los datos recogidos antes y después del programa de formación en DIT, incorporando la AT como variable independiente (VI) cuyos datos se recogieron de la siguiente manera:
Al grupo experimental, se aplicó una lista de cotejo del desempeño e incorporó la VI, a través de tres visitas de asistencia técnica: (M1) antes del programa de formación en DIT, (M2) durante la formación y (M3) al finalizar el proceso de formación.

Al grupo control, se aplicó la lista de cotejo del desempeño que recoge información en dos momentos: antes y al final del programa de formación en DIT para observar y comparar su nivel de avance.

\subsection{Instrumentos}

Durante la investigación se elaboraron dos instrumentos. Para la variable dependiente, se contó con una Guía para el facilitador; y para la variable independiente, se formuló una lista de cotejo, que fue sometida a un proceso de consulta de jueces para la validación de contenido y confiabilidad del instrumento.

Para el proceso de validez se realizó el análisis de concordancia de opiniones de expertos, para ello se utilizó la prueba de Aiken observando concordancia en las opiniones, con un nivel de confianza del $95 \%$ y margen de error de $5 \%$. Además, se determinó que el instrumento presentó alta confiabilidad, de acuerdo al valor calculado de Alfa de Cronbanh, con una confiabilidad es aceptable, siendo mayor a 0.80 .

La lista de cotejo contiene 58 ítems organizadas en cinco dimensiones del desempeño seleccionadas para esta investigación: (1) Organiza el espacio para promover DIT en el Control de CRED, (2) Organiza recursos y materiales para promover DIT, (3) Interacciones del personal de salud con el niño o niña durante la atención en el Control de CRED, (4) Evalúa el desarrollo infantil en el Control de CRED considerando las necesidades y condiciones físicas y afectivas del niño/a, (5) Consejería a la familia durante la atención en el Control de CRED. 
El instrumento fue aplicado por el equipo capacitador del programa de formación en DIT a través de visitas a cada enfermera en los establecimientos de salud donde brindan el servicio de CRED. Además, se aplicó el principio de autonomía y decisión de los participantes a través de la aplicación del consentimiento informado.

\section{Resultados}

Tabla 1

Comparación del desempeño global, entre el grupo experimental y control, luego de la aplicación de la asistencia técnica.

\begin{tabular}{|l|c|c|c|c|c|}
\hline & \multicolumn{4}{|c|}{ Grupos } & \\
\hline & \multicolumn{2}{|c|}{$\begin{array}{c}\text { Experimental } \\
(\mathrm{N}=15)\end{array}$} & \multicolumn{2}{c|}{ Control $(\mathrm{N}=15)$} & \\
\hline & $\mathrm{M}$ & $\mathrm{DE}$ & $\mathrm{M}$ & $\mathrm{DE}$ & $\mathrm{u}$ \\
\hline $\begin{array}{l}\text { Desempeño } \\
\text { global }\end{array}$ & 51.67 & 3.13 & 26.53 & 11.30 & $.500^{* *}$ \\
\hline
\end{tabular}

${ }^{* *} P \leq .01$

La tabla 1 muestra diferencias significativas entre el grupo experimental y el grupo control. El grupo experimental, alcanzó una media del 51.67 mientras que el grupo control solo llegó a 26.53, aplicando la prueba no paramétrica U Mann Whitney: $p \leq .01$. La formación en DIT genera avances en ambos grupos, pero al incorporar la AT se evidencia un salto significativo respecto al grupo control.

Tabla 2

Comparación en: Organiza el espacio para promover Desarrollo Infantil Temprano en el control de CRED, luego de la aplicación de la asistencia técnica.

\begin{tabular}{|l|c|c|c|c|c|}
\hline \multirow{2}{*}{} & \multicolumn{4}{|c|}{ Grupos } & \\
\cline { 2 - 7 } & \multicolumn{2}{|c|}{$\begin{array}{c}\text { Experimental } \\
(\mathrm{N}=15)\end{array}$} & \multicolumn{2}{|c|}{$\begin{array}{c}\text { Control } \\
(\mathrm{N}=15)\end{array}$} & \\
\cline { 2 - 7 } & $\mathrm{M}$ & $\mathrm{DE}$ & $\mathrm{M}$ & $\mathrm{DE}$ & $\mathrm{u}$ \\
\hline $\begin{array}{l}\text { Acondiciona el espacio para la atención del } \\
\text { desarrollo en el Control de CRED }\end{array}$ & 3.73 & .458 & 2.00 & 1.36 & $27.000^{* *}$ \\
\hline $\begin{array}{l}\text { Dispone de mobiliario adecuado y suficiente } \\
\text { para evaluar y promover el desarrollo }\end{array}$ & 2.73 & .799 & 1.20 & 1.21 & $33.500^{* *}$ \\
\hline $\begin{array}{l}\text { Cuenta adicionalmente con espacios que } \\
\text { permiten promover el DIT con familias, } \\
\text { niños y niñas. }\end{array}$ & 1.53 & .640 & .87 & .83 & $62.500^{*}$ \\
\hline $\begin{array}{l}\text { Organiza el espacio para promover } \\
\text { Desarrollo Infantil Temprano (DIT) en el } \\
\text { Control de CRED }\end{array}$ & 8.00 & 1.51 & 4.07 & 2.96 & $28.00^{* *}$ \\
\hline
\end{tabular}

${ }^{*} P \leq .05$

${ }^{* *} P \leq .01$ 
En la tabla 2, al comparar este desempeño se puede observar que el grupo experimental alcanzó una media de 8, mientras que en el grupo control solo alcanzó una media de 4.07, siendo esto significativo al $\mathrm{p} \leq .01$.

Si bien se observa que todos los indicadores de esta dimensión muestran diferencias significativas entre el grupo ex- perimental y el grupo control, los mejores avances están en los indicadores: acondiciona el espacio para la atención del desarrollo del CRED y dispone de mobiliario adecuado y suficiente para evaluar y promover el DIT. Al observar estos resultados se evidencian mejoras en su capacidad para organizar espacios diferenciados para la atención del CRED.

\section{Tabla 3}

Comparación en: Organiza recursos y materiales para promover DIT, luego de la aplicación de la asistencia técnica.

\begin{tabular}{|l|c|c|c|c|c|}
\hline \multirow{2}{*}{} & \multicolumn{4}{|c|}{ Grupos } & \\
\cline { 2 - 7 } & \multicolumn{2}{|c|}{$\begin{array}{c}\text { Experimental } \\
(\mathrm{N}=15)\end{array}$} & \multicolumn{2}{|c|}{$\begin{array}{c}\text { Control } \\
(\mathrm{N}=15)\end{array}$} & \\
\cline { 2 - 7 } & $\mathrm{M}$ & $\mathrm{DE}$ & $\mathrm{M}$ & $\mathrm{DE}$ & $\mathrm{u}$ \\
\hline $\begin{array}{l}\text { Organiza materiales pertinentes para la } \\
\text { evaluación y seguimiento del desarrollo. }\end{array}$ & 2.73 & .458 & 1.47 & .834 & $23.500^{* *}$ \\
\hline $\begin{array}{l}\text { Dispone juguetes y recursos que } \\
\text { responden al interés lúdico de los niños } \\
\text { y niñas y promueven su desarrollo. }\end{array}$ & 4.27 & 1.10 & 1.40 & 1.29 & $13.000^{* *}$ \\
\hline $\begin{array}{l}\text { Mantiene juguetes y materiales en } \\
\text { adecuadas condiciones de conservación } \\
\text { y uso. }\end{array}$ & 2.00 & .000 & 1.80 & .561 & 97.500 \\
\hline $\begin{array}{l}\text { Organiza recursos y materiales para } \\
\text { promover DIT }\end{array}$ & 9.00 & 1.36 & 4.67 & 2.19 & $9.00^{* *}$ \\
\hline
\end{tabular}

${ }^{* *} P \leq .01$

En la tabla 3 se observa que las diferencias en el puntaje general de este desempeño son significativas. El grupo experimental mostró una media de 9, mientras que el grupo control llegó a 4.6, siendo esto significativo al $p \leq .01$.

En cuanto a los indicadores en esta dimensión, se observa significancia en la capacidad para disponer juguetes y recursos que responden al interés lúdico de los niños y niñas, así como a su capacidad para organizar materiales pertinentes para la evaluación y seguimiento del desarrollo. Sin embargo, en el indicador relacionado al mantenimiento de juguetes y materiales en adecuadas condiciones de conservación y uso, se observa menos avance. 
Tabla 4

Comparación en: Interacciones del personal de salud con el niño o niña durante la atención en el Control de CRED, luego de la aplicación de la asistencia técnica.

\begin{tabular}{|l|c|c|c|c|c|}
\hline & \multicolumn{4}{|c|}{ Grupos } & \\
\hline & \multicolumn{2}{|c|}{$\begin{array}{c}\text { Experimental } \\
(\mathrm{N}=15)\end{array}$} & \multicolumn{2}{|c|}{$\begin{array}{c}\text { Control } \\
(\mathrm{N}=15)\end{array}$} & \\
\hline & $\mathrm{M}$ & $\mathrm{DE}$ & $\mathrm{M}$ & $\mathrm{DE}$ & $\mathrm{u}$ \\
\hline $\begin{array}{l}\text { Brinda atención al niño o niña respetando } \\
\text { las necesidades individuales y afectivas. }\end{array}$ & 7.00 & .000 & 4.00 & 1.195 & $.000^{* *}$ \\
\hline $\begin{array}{l}\text { La atención es pertinente a las necesidades } \\
\text { lúdicas y culturales del niño o niña. }\end{array}$ & 3.93 & .258 & 2.87 & 1.125 & $49.500^{* *}$ \\
\hline $\begin{array}{l}\text { Promueve autonomía y libertad de } \\
\text { movimiento en la atención al niño o niña. }\end{array}$ & 2.00 & .000 & .67 & .900 & $30.000^{* *}$ \\
\hline $\begin{array}{l}\text { Interacciones del personal de salud con el niño o } \\
\text { niña durante la atención en el Control de CRED }\end{array}$ & 12.93 & 2.58 & 7.53 & 2.825 & $.000^{* *}$ \\
\hline
\end{tabular}

${ }^{* *} P \leq .01$

En la tabla 4, se observa mejores resultados en el grupo experimental con una media de 12.93 , mientras que el grupo control alcanzó 7.53 , siendo esto significativo al $p \leq .01$.

Los desempeños de interacción fueron los que mostraron mejores avances. Si bien la capacitación contribuye al logro de este desempeño, se evidencian diferencias significativas al incorporar AT. La mayoría demostraba una interacción positiva, llamando a niñas o niños por su nombre, saludándolos, estableciendo contacto visual, explicando, anticipando, pidiendo permiso al realizarles los procedimientos y hablando en su lengua materna.

Tabla 5

Comparación en: Evalúa el desarrollo infantil temprano en el Control de CRED considerando las necesidades y condiciones físicas y afectivas del niño/a.

\begin{tabular}{|l|c|c|c|c|c|}
\hline \multirow{2}{*}{} & \multicolumn{4}{|c|}{ Grupos } & \\
\cline { 2 - 6 } & \multicolumn{2}{|c|}{$\begin{array}{c}\text { Experimental } \\
(\mathrm{N}=15)\end{array}$} & \multicolumn{2}{|c|}{$\begin{array}{c}\text { Control } \\
(\mathrm{N}=15)\end{array}$} & \\
\cline { 2 - 6 } & $\mathrm{M}$ & $\mathrm{DE}$ & $\mathrm{M}$ & $\mathrm{DE}$ & $\mathrm{u}$ \\
\hline $\begin{array}{l}\text { Aplica estrategias de evaluación del } \\
\text { desarrollo en el Control de CRED. }\end{array}$ & 6.80 & .414 & 3.00 & 1.92 & $10.500^{* *}$ \\
\hline $\begin{array}{l}\text { Registra y comunica los resultados de la } \\
\text { evaluación del desarrollo. }\end{array}$ & 3.00 & .000 & 2.00 & .655 & $22.500^{* *}$ \\
\hline $\begin{array}{l}\text { Evalúa el desarrollo infantil temprano } \\
\text { en el Control de CRED considerando } \\
\text { las necesidades y condiciones físicas y } \\
\text { afectivas del niño/a. }\end{array}$ & 9.80 & .415 & 5.00 & 2.39 & $9.000^{* *}$ \\
\hline
\end{tabular}

${ }^{* *} P \leq .01$ 
En la tabla 5 se observa que el grupo experimental alcanzó una media de 9.8, siendo esto significativo al $p \leq .01$, mientras que el grupo control alcanzó una media de 5 .

Se observa que en su mayoría aplican el Test Abreviado Peruano, evaluando los hitos del desarrollo para la edad. El aspecto menos logrado es el registro de los resultados en la Tarjeta de control CRED, así como informar a la familia sobre los avances en el desarrollo. Muchas veces se informa solo los resultados del estado nutricional.

\section{Tabla 6}

Comparación en: Consejería a la familia durante la atención en el Control de CRED, luego de la aplicación de la asistencia técnica.

\begin{tabular}{|c|c|c|c|c|c|}
\hline & \multicolumn{4}{|c|}{ Grupos } & \multirow[b]{3}{*}{$\mathrm{u}$} \\
\hline & \multicolumn{2}{|c|}{$\begin{array}{l}\text { Experimental } \\
\quad(\mathrm{N}=15)\end{array}$} & \multicolumn{2}{|c|}{ Control $(\mathrm{N}=15)$} & \\
\hline & M & $\mathrm{DE}$ & M & $\mathrm{DE}$ & \\
\hline $\begin{array}{l}\text { Muestra empatía con la familia al } \\
\text { comunicar mensajes. }\end{array}$ & 3.93 & .258 & 2.40 & 1.454 & $34.500^{* *}$ \\
\hline $\begin{array}{l}\text { Orienta a las familias brindando una } \\
\text { consejería pertinente a las necesidades } \\
\text { del niño o niña y la familia. }\end{array}$ & 6.27 & .594 & 2.07 & 1.486 & $5.500^{* *}$ \\
\hline $\begin{array}{l}\text { Promueve la formulación de compromisos } \\
\text { con la participación de las familias. }\end{array}$ & 1.73 & .458 & .80 & .941 & $51.500^{* *}$ \\
\hline $\begin{array}{l}\text { Consejería a la familia durante la atención } \\
\text { en el Control de CRED }\end{array}$ & 11.93 & .799 & 5.27 & 3.218 & $8.000^{* *}$ \\
\hline
\end{tabular}

${ }^{* *} P \leq .01$

En la tabla 6 se observa que el grupo experimental alcanzó una media de a 11.93, lo cual representa un avance significativo al $p \leq .01$, mientras que el grupo control mostró una media de 5.27.

La capacidad del personal de salud para brindar consejería en DIT es un aspecto complejo. Al inicio, en su mayoría centraba la información en contenidos nutricionales o de crecimiento, pero no siempre sobre los hitos del desarrollo. Si bien el estado nutricional es muy importante, se observa que es necesario complementarlo con aspectos relacionados al desarrollo.

\section{Discusión}

Al inicio de la investigación ambos grupos parten de condiciones similares y no se evidencian diferencias significativas entre el desempeño global y en los componentes observados en el grupo experimental y el de control. Contar con grupos parejos en el punto de partida de la investigación nos permite mostrar las diferencias al incorporar la variable asistencia técnica.

La asistencia técnica como elemento complementario a los programas de formación continua genera un efecto significativo en el desempeño profesional y ello contribuye con la calidad de los servicios que brindan.

El estudio pone en evidencia que la formación académica por sí misma puede resultar insuficiente para un adecuado desempeño. La asistencia técnica, agrega valor y calidad a los programas de formación. De acuerdo con lo señalado por Teje- 
dor (2019) y Salas-Perea et al. (2016), el desempeño del personal de salud no solo depende de la formación académica, sino especialmente de la capacidad de actuar e intervenir en la atención directa con el usuario. En tal sentido, es necesario enfocarnos en estrategias de formación orientadas a mejorar el desempeño profesional y no solo en la acumulación o actualización de información.

Los resultados coinciden con la evidencia vinculada a experiencias del sector educación, que han mostrado el efecto positivo del acompañamiento pedagógico a los docentes. De acuerdo con Leiva-Guerrero y Vásquez (2019) citan a Barber y Mourshed (2008), para explicar el efecto del acompañamiento al docente desde la perspectiva del liderazgo pedagógico y su fortalecimiento en la práctica. Precisan que ello representa una variable de impacto en la eficacia y mejora de las capacidades y transformación de las escuelas.

En tal sentido, el desempeño docente es mejor cuando se promueven espacios de entrenamiento en la práctica y cuando se facilita la retroalimentación desde el análisis crítico-reflexivo. Este fue un aspecto tomado en cuenta en la asistencia técnica, el cual sigue un proceso ordenado y sistemático de observación, ejercitación y reflexión sobre la atención en el CRED, propiciando que el personal de salud, tenga espacios de autoreflexión sobre su práctica, toma de decisiones para promover cambios, que generan un efecto en la mejora de la calidad de la atención que brindan.

Datos similares coinciden con la experiencia de AT Cooperación y Acompañamiento Territorial (Minsalud, 2014), muestra que los procesos del AT mejoran las capacidades individuales, organizacionales y comunitarias, contribuyendo al logro de la gestión territorial para alcanzar los resultados esperados. Por otro lado, Salas-Perea et al. (2016) afirman que la eficiencia en el desempeño está unida e integrada a la calidad de los servicios.

La investigación pudo determinar que existen diferencias significativas que favorecen al grupo experimental, mostrando que la AT en un programa de formación continua, mejora el desempeño profesional. El personal de salud que formó parte del grupo experimental mostró mejoras en las cinco dimensiones del desempeño, que representan los aspectos más relevantes en la atención del desarrollo en el Control de CRED: (1) capacidad para acondicionar el espacio, (2) capacidad para organizar recursos y materiales, (3) capacidad para mantener interacciones positivas con niños y niñas, (4) capacidad para aplicar la evaluación de desarrollo y (5) capacidad para brindar consejería a la familia en DIT.

Los aspectos que alcanzaron mejores resultados fueron la capacidad para organizar juguetes y materiales, así como establecer interacciones positivas con el niño o niña. El aspecto más complejo de avanzar fue el referido a la capacidad para organizar espacios y mobiliario adecuado, el cual requería procesos de gestión, cuyas acciones en el Estado suelen ser largos y complicados, además de resistencias en el mismo personal para generar modificaciones en sus espacios o una nueva organización. Las condiciones para la atención educativa, social o de salud, representan un aspecto en la que el personal cumple un rol clave, ya que organiza el espacio, toma decisiones en la ambientación y selección de material para una atención adecuada. Al respecto, el estudio de Cordero et al. (2016) muestra que las condiciones del espacio y equipamiento son aspectos que evidencian calidad en el servicio y están vinculados a la capacidad de gestión del personal de salud.

Al inicio del programa se pudo observar que en su mayoría las enfermeras tenían dificultad para organizar espacios dife- 
renciados que faciliten las actividades de CRED en atención del niño o niña. Muchas veces solo contaban con espacios para evaluar el estado nutricional y no con las condiciones para observar el desarrollo o sus espacios eran limitados, con insuficiente mobiliario y recarga visual.

En la última visita se observaron mejoras en los ambientes, los cuales evidencian espacios y condiciones específicas para evaluar el crecimiento y el desarrollo de manera equitativa. Los espacios se encontraban sin interferencias o recarga visual, con información utilitaria sobre DIT en la sala de espera o el consultorio. Además, se observó el uso de organizadores para los materiales o juguetes, soporte firme y blando en el piso para favorecer posturas y desplazamientos, y en algunos casos, mejoras en la sala de espera, acondicionada para recibir a niñas, niños y sus familias.

En el grupo experimental se observan cambios en los espacios, mostrando recursos o condiciones básicas para promover el DIT, favorecer actividades cotidianas como el cambio de ropa, la lactancia o mobiliario apropiado para evaluar a los niños de 2 y 3 años. Incluso, en algunos casos, se evidencia un espacio dispuesto para realizar sesiones con familias, los cuales demandaron gestiones, adecuaciones o procesos de adquisición. Estos aspectos muchas veces requieren de mayor tiempo para completar la gestión que permita contar con mobiliarios o realizar la reubicación de espacios. Sin embargo, se observó que en la mayoría del grupo experimental lograron conseguirlos o estaban en procesos de gestión. En cada aspecto de este desempeño, se observan diferencias entre el grupo experimental y control.

En cuanto a la capacidad para organizar recursos y materiales, se pudo observar que en ambos casos mejoró el desempeño al incluir recursos y materiales lúdicos para el DIT. Sin embargo, se evidencia mejores logros y avance en el grupo experimental en su capacidad para organizar juguetes y materiales para promover el DIT y que respondan al interés lúdico de niñas y niños. El mayor reto para el personal de salud en este aspecto fue contar con juguetes elaborados con material del contexto o que represente un referente accesible para las familias, así como mantener los juguetes y materiales en adecuadas condiciones de conservación y uso.

Un aspecto relevante fue evidenciar que al inicio no había un equilibro entre las acciones orientadas a promover el crecimiento, con las de desarrollo. En su mayoría tenían los implementos de antropometría para evaluar peso y talla, pero no los instrumentos o condiciones para evaluar los hitos del desarrollo psicomotor, cognitivo, vínculo de apego, comunicación o exploración. Al final de la experiencia, todos los participantes del grupo experimental lograron implementar las condiciones físicas y materiales para promover el DIT en sus consultorios.

En cuanto a la dimensión sobre la capacidad para mantener interacciones positivas con niños y niñas, se observó que el grupo experimental alcanza un mejor desempeño. La calidad de las interacciones tiene un gran impacto en la vida de los niños y niñas en edades tempranas y es una dimensión en la que el personal de salud debe ser referente para las familias.

De acuerdo con lo señalado por López et al. (2016), las interacciones entre los cuidadores y los niños, es uno de los aspectos críticos en la calidad de los servicios de atención infantil, que se expresa en la capacidad de respuesta y la sensibilidad de los cuidadores hacia los niños y niñas. Este aspecto es muy importante en el desarrollo de los bebés o niños pequeños pues tiene un impacto de largo plazo. 
Las evidencias de la serie The Lancet, (Unicef et al., 2016; Unicef et al., 2018) destacan la importancia de un cuidado cariñoso y sensible en este periodo de vida. Señala que la calidad de las interacciones tiene un efecto positivo, no solo en el desarrollo físico, emocional, social y cognitivo, sino que protege a los niños y niñas pequeños/as de la adversidad, produciendo un efecto a lo largo de la vida.

Monteiros (2015) menciona que la atención con calidez, el trato al usuario y la sensibilidad de los profesionales es uno de los aspectos de proceso que define la satisfacción del usuario en los centros de salud familiar. Concluye que la manera "seca" o "tajante" de algunos profesionales hacia el usuario, el niño o niña durante el control de salud, influye en la opinión del acompañante. Por su parte, Fernández (2017) afirma que los aspectos interpersonales, técnico asistencial y capacidad educadora, son necesarios para un adecuado desempeño y forman una opinión favorable de la imagen del personal de salud en las madres de familia.

El sector salud ha venido generando procesos de cambios normativos coherentes con los enfoques de derechos y de atención integral que responden al marco global del cuidado cariñoso y sensible. Ello requiere asumir nuevos paradigmas $y$ desarrollar habilidades personales para interactuar con los niños y niñas y con sus familias. Estos aspectos no solo suponen la adquisición de nueva información, es necesario generar espacios de reflexión, análisis, pero también de ejercitación de nuevas habilidades y actitudes. De allí que la asistencia técnica en el campo profesional representa una estrategia que contribuye en la mejora del desempeño y demanda nuevos retos en los procesos de formación continua.

En las interacciones y trato al niño, mostraron cambios importantes; sin embargo, el aspecto que aún representa un reto para el personal de salud es el de promover la autonomía y libertad de movimiento durante la atención de CRED. Si bien hay diferencias entre ambos grupos y mejores niveles de avance en el grupo experimental, en ambos casos se observa dificultad para generar espacios de libre expresión; hay una tendencia a dirigir el juego del niño o niña.

En la dimensión sobre la capacidad para aplicar los instrumentos de evaluación del desarrollo, se observa que el grupo experimental alcanza un mejor desempeño. Fernández (2017) afirma que la evaluación del desarrollo es una de las actividades del personal de enfermería que requiere entrenamiento, especialmente para las habilidades de observación y manejo de los instrumentos, para tomar acciones oportunas de derivación y seguimiento en el desarrollo.

Al aplicar los instrumentos de evaluación, se observó que el personal de salud, en su mayoría, no contaba con las condiciones y recursos adecuados para realizar esta actividad. Si bien el grupo experimental evidencia mayores cambios en este aspecto, se observó que uno de los retos en ambos grupos, era aplicar el procedimiento de iniciar la evaluación con los hitos del mes anterior, para verificar niveles de logro y retrasos en el desarrollo (como indica el instrumento de evaluación). Al inicio, la mayoría tampoco observaba los indicadores de desarrollo a partir del juego libre o por propia iniciativa. Luego, ambos grupos fueron ejercitando este aspecto y afinando su mirada durante la evaluación.

Lo observado en este aspecto coincide con lo sustentado por el Ministerio de Salud de Argentina (2017) sobre la evaluación del desarrollo, explican que requiere de personal de salud con habilidades y actitudes para la observación, con capacidad para mirar al niño, desde sus carac- 
terísticas, como sujeto de derechos, con potencial y con iniciativa, que respete sus tiempos, proceso y contexto.

En la dimensión sobre la capacidad para brindar consejería a la familia en DIT, se observó que el grupo experimental alcanzó un mejor desempeño respecto al grupo Control. De acuerdo con lo señalado por Guerrero (2019) citan a Siraj-Blatchford y Woodhead (2009), para describir los desafíos de la calidad de los programas de primera infancia, afirman que para ser eficaces requieren de profesionales capacitados y asistidos que promuevan la participación de las familias, quienes deben ser también asistidas y recibir una adecuada consejería que les permita mejorar sus prácticas de crianza.

Por otro lado, Cordero et al. (2016), al evaluar la consejería, pone énfasis en la necesidad del entrenamiento y la práctica de habilidades interpersonales y de comunicación. Para ello, incorpora metodologías demostrativas y pertinentes al contexto que mejoran las prácticas de cuidado infantil.

En esta dimensión, el aspecto más difícil de alcanzar fue la capacidad de promover compromisos expresados espontáneamente por las familias. Muchas veces, la tendencia era dar consejos y recomendaciones en la que les decían lo que debían hacer, antes de promover que sean las mismas familias, quienes, a partir de un proceso de reflexión con el personal de enfermería, se den cuenta y propongan sus propios compromisos.

Finalmente, un aspecto relevante que contribuyó con la mejora de la consejería y que fue valorado por el personal de salud, fue el tipo de asistencia técnica que recibieron. Muchas de las participantes expresaron que el acompañamiento recibido les resultó un referente positivo para mejorar las consejerías a las familias. A partir del análisis y toma de decisiones de las mis- mas enfermeras durante la AT, se daban cuenta que también podían orientar una consejería centrada en el protagonismo de las mismas familias.

\section{Conclusiones}

Se determinó que la asistencia técnica mejora el desempeño del personal de salud que atienden en el Control de CRED, fortaleciendo e incrementando sus capacidades.

La asistencia técnica contribuye, complementa y hace más eficiente los programas de formación continua de los profesionales de salud que atienden en el Control de CRED, generando mejoras en su desempeño. Los resultados muestran diferencias significativas al incorporar la asistencia técnica en un programa de formación: el grupo experimental alcanzó una media de 51.67, frente al grupo control que llegó a 26.53.

\section{Recomendaciones}

La AT debe complementar e ir en paralelo al proceso de capacitación, con visitas de inicio, proceso y término. Esto permite, tener claro el punto de partida de cada participante; luego, conocer y acompañar al personal de salud durante la formación; $y$, finalmente, identificar cómo terminó su desempeño para brindar los refuerzos que sean necesarios y cerrar sus procesos de aprendizaje.

En un programa de formación, es recomendable, que quienes realizan las visitas de asistencia técnica sean parte del equipo de capacitación, para complementar y retroalimentar los aprendizajes del programa de formación. Así los participantes conectan la teoría con la práctica, encuentran más sentido a sus aprendizajes y se instalan con mayor efectividad en su desempeño cotidiano.

Es recomendable que las coordinadoras de Red o supervisoras del área de salud 
sean parte del equipo formador o acompañen a las formadoras en las visitas de AT. La participación del personal supervisor de las instancias regionales o locales de salud en las visitas de asistencia técnica fue fundamental para el desarrollo de esta experiencia, generó oportunidades para una mayor sostenibilidad de la metodología en los procesos de formación y asistencia técnica.

La AT puede vincularse a resultados en el niño o niña, sus familias y a la calidad del servicio. Se recomienda que, al igual que en educación, se generen investigaciones que vinculen la relación entre el desempeño del personal de salud con los resultados en el desarrollo infantil temprano en los usuarios, ya sea en los mismos niños y niñas, como en las prácticas positivas de crianza de las familias.

En el mismo sentido, sería recomendable generar evidencias sobre la relación entre la mejora en el desempeño del personal de salud y la calidad de los servicios. Las políticas públicas orientan una lógica centrada en resultados y no solo en procesos, por ello sería recomendable que la investigación en los temas de desempeño profesional pueda continuar y avanzar en la búsqueda de los efectos en el desarrollo infantil temprano y en la calidad en los servicios de salud.

Generar una propuesta de AT integral que incluya desempeños vinculados a la atención en crecimiento y desarrollo. La investigación centró la mirada en los contenidos del desempeño relacionados al DIT, para superar vacíos y diferencias que existían entre las acciones de estado nutricional y desarrollo, equilibrando así la atención de ambos procesos en el control de CRED. Sin embargo, consideramos que, a partir de esta investigación, existen suficientes elementos para impulsar una propuesta integral que incluya contenidos de nutrición y desarrollo en el desempeño del profesional de salud.

\section{Referencias bibliográficas}

Balarin, M., \& Escudero, A. (2019). El acompañamiento pedagógico como estrategia de formación docente en servicio: Reflexiones para el contexto peruano Serie: Aportes para la reflexión y construcción de políticas docentes (Grupo de Análisis para el Desarrollo (GRADE), Ed.; UNESCO). https://unesdoc.unesco.org/

Cordero, L., Montes, C., Velásquez, J., Rodríguez, Y., Vigo, W., \& Rosas-Aguirre, A. (2016). Estudio Cuali-Cuantitativo sobre la atención de salud de niños menores de tres años en establecimientos de salud de nueve regiones pobres del Perú. Revista Peruana de Medicina Experimental y Salud Publica, 33(1), 21-31. https://doi. org/10.17843/rpmesp.2016.331.1922

Fernández, R. (2017). UNIVERSIDAD NACIONAL MAYOR DE SAN MARCOS Opinión sobre la imagen del profesional de enfermería de las madres de familia que acuden al servicio de Crecimiento y Desarrollo del Centro de Salud Zárate 2016.

Guerrero, G. (2019). Perú: Informe de progreso de políticas de Primera Infancia. https://bit.ly/33Ne0mq

Leiva-Guerrero, M., \& Vásquez, C. (2019). Liderazgo pedagógico: De la Supervisión al acompañamiento docente. http://dx.doi. org/10.31619/caledu.n51.635

López, F., Araujo, C., \& Tomé, R. (2016). ¿Cómo se mide la calidad de los servicios de cuidado infantil? Guía de herramientas (BID). https://publications.iadb.org/publications/spanish/document/\%C2\%BFC\%C3\%B3mo-se-mide-la-calidad-de-los-servicios-de-cuidado-infantil-Gu\%C3\%ADa-de-herramientas.pdf

Ministerio de Educación [Minedu]. (2016). Norma técnica para el acom- 
pañamiento pedagógico en la EBR. In RSG-N008-2016-MINEDU. https://www. gob.pe/institucion/minedu/normas-legales/123819-008-2016-minedu

Ministerio de Educación [Minedu]. (2014). Programa de Educación "Logros de Aprendizaje de los Estudiantes de Educación Básica Regular - PELA 2013- 2016”. EI acompañamiento pedagógico. Protocolo del Acompañante Pedagógico, del Docente Coordinador/ Acompañante y del formador. Lima, Perú.

Ministerio de Salud de Argentina. (2017). Criterios para la aplicación del Instrumento de Observación del Desarrollo Infantil (IODI) (Presidencia de la Nación y programa de Cobertura Universal de salud de Argentina., Ed.). https://bancos.salud.gob.ar/sites/default/files/201810/0000001082cnt-guia-iodi-dic-2017.pdf Ministerio de Salud [Minsa]. (2017). Norma Técnica de Salud para el Control de Crecimiento y Desarrollo de la niña y niño menores de cinco años. In NTS $N^{\circ}$ 137-MINSA/2017/DIGIESP. https:// cdn.www.gob.pe/uploads/document/file/191049/537-2017-MINSA.pdf

Minsalud. (2014). Guía operativa Asistencia técnica, cooperación y acompañamiento territorial-ACAT-, para la implementación de la Atención Integral en Salud a la primera infancia, infancia y la adolescencia Dirección de Promoción y Prevención Grupo Curso de Vida. https://www.minsalud.gov.co/sites/rid/Lists/BibliotecaDigital/ RIDE/VS/PP/Guia-asistencia-tecnica.pdf

Monteiros, F. (2015). Satisfacción usuaria con el control de salud infantil en un Centro de Salud familiar en el año 2015. http:// bibliodigital.saludpublica.uchile.cl:8080/ handle/123456789/381

Morales, P. (2012). Tipos de variables y sus implicaciones en el diseño de una investigación. Madrid: Universidad Pontificia Comillas.
Neill, D., \& Cortez, L. (2018). Procesos y Fundamentos de la Investigación Científica (Colección REDES 2017). http://repositorio.utmachala.edu.ec/bitstream/48000/12498/1/Procesos-y-FundamentosDeLainvestiagcionCientifica.pdf

Otzen, T., \& Manterola, C. (2017). Técnicas de Muestreo sobre una Población a Estudio Sampling Techniques on a Population Study. Int. J. Morphol, 35(1), 227232. https://scielo.conicyt.cl/pdf/ijmorphol/ v35n1/art37.pdf

Rodríguez, J., Leyva, J., \& Hopkins, A. (2016). El efecto del Acompañamiento Pedagógico sobre los rendimientos de los estudiantes de escuelas públicas rurales del Perú - Informe Final. http://umc.minedu.gob.pe/?cat=9.

Salas-Perea, R., Quintana_Galede, M., \& Pérez-Hoz, G. (2016). Formación basada en competencias en ciencias de la salud. MediSur (Revista En Internet), 14(4), 456463. http://www.medisur.sld.cu/index.php/ medisur/article/view/3363

Salluca, M., \& Valeriano, H. (2018). Acompañamiento pedagógico y el rol docente en jornada escolar completa: Caso instituciones educativas Santa Rosa y Salesianos de San Juan Bosco - Puno Perú. Revista de Investigaciones Altoandinas - Journal of High Andean Research, 20(1), 137148. https://doi.org/10.18271/ria.2018.337

Solís, S., Pupo, Y., Rodríguez, A., Silenia, V., Olivares, G., \& López, A. (2019). Competencias y desempeño profesional desde la educación médica. Revista Cubana de Tecnología de La Salud, 10(1). www.revtecnología.sld.cu

Tejedor, V. E. (2019). La evaluación del desempeño incrementa la calidad en la atención en las empresas de servicios. Revista FAECO Sapiens, 2(2). http://portal.amelica.org/ameli/jatsRepo/221/221970021/ index.html 
Torres-López, S., Cuesta-Santos, A., Yobanis Piñero-Pérez, P., \& Alejandro Lugo-García, J. (2017). Evaluación de competencias laborales a partir de evidencias. Ingeniería Industrial, XXXIV(2), 124-134. http://www.rii.cujae.edu.cu

UNICEF, World Bank Group, World Heatlh Organization, \& ECDAN, T. P. E. W. E. C. (2018). Resumen Ejecutivo: Cuidado cariñoso y sensible para el desarrollo en la Primera Infancia. www.nurturing-care.org
UNICEF, World Bank Group, World Heatlh Organization, \& ECDAN, T. P. y E. W. E. C. (2016). Apoyando el desarrollo en la primera infancia de la ciencia a la aplicación a gran escala. Un resumen ejecutivo de la serie de The Lancet. http://repositorio.minedu.gob.pe/handle/MINEDU/5993 